\title{
Estudo das interações medicamentosas em prescrições de pacientes de uma Clínica-Escola de Fisioterapia
}

\author{
Study of drug interactions in prescriptions for patients in a Clinic-School of \\ Physiotherapy
}

Recebido em: 29/10/2015

Aceito em: $\quad 28 / 12 / 2015$

\author{
Jean Charles POPETS; Gustavo Batista ANDRADE; \\ Alessandra STROKA; Sandro ROSTELATO-FERREIRA \\ Universidade Paulista, Campus Sorocaba. Av. Independencia 210, Éden, \\ Sorocaba, SP. CEP 18087-101, Brasil.E-mail: sandrorostelato@yahoo.com.br
}

\begin{abstract}
Most of the patients with chronic diseases use many drugs are known as polypharmacotherapy. The latter being individuals who have a higher chance of suffering harmful effects of drug interactions, which did not fail to be different with patients of Physiotherapy School Clinic at Sorocaba city. The aim of this study was to investigate the evidence of the prescriptions of patients at the Clinical School of Physiotherapy. The prescriptions of patients in physiotherapy treatment, in the Neurology and Orthopedics areas, were analyzed. The results were obtained from a broad range of drugs currently used in patients presenting with from 1 to 14 drug interactions with other drugs. The most commonly used drugs were in cardiology, with a high prevalence of losartan and hydrochlorothiazide associations, present in $28.57 \%$ of the analyzed prescriptions. Hypertension, in conjunction with diabetes, causes a worsening of health and increases of drugs to be used in these patients. It is concluded that the primary interactions presented on the requirements of patients Clinical Physiotherapy School, at Sorocaba city, occurred among antihypertensive classes, nonsteroidal anti-inflammatory steroids, and hypoglycemic, confirming the need for the pharmacist to order the rational use of drugs
\end{abstract}

Keywords: drug-drug interaction; prescription; Physiotherapy; Kinesiotherapy

\section{RESUMO}

Pacientes portadores de doenças crônicas são, em sua maioria, usuários de uma grande quantidade de medicamentos, o que configura polifarmacoterapia. Estes indivíduos apresentam maior possibilidade de sofrerem efeitos nocivos das interações medicamentosas e isso foi tem sido observado em pacientes de uma Clínica-Escola de Fisioterapia no município de Sorocaba. O objetivo desse trabalho foi investigar evidências de interação medicamentosa em prescrições médicas de pacientes da Clínica Escola de Fisioterapia. Foram analisadas as prescrições médicas dos pacientes da área de Neurologia e ortopedia em tratamento de fisioterapia. Os resultados obtidos foram de uma grande variedade de medicamentos em uso por esses pacientes, apresentando de uma até 14 interações entre os medicamentos utilizados. Os fármacos mais utilizados foram na área de cardiologia, com elevada predominância de associações de losartana e hidroclorotiazida, estando presentes em $28,57 \%$ das prescrições analisadas. A hipertensão, em conjunto com o diabetes, causa a piora da saúde, aumentando a necessidade de medicamentos a serem usados nestes pacientes. Com base nos resultados foi possível concluir que as principais interações apresentadas nas prescrições dos pacientes da Clínica-Escola de Fisioterapia, no município de Sorocaba, SP, ocorreram entre as classes de anti-hipertensivos, anti-inflamatórios não-esteroidais e hipoglicemiantes, ratificando a necessidade do profissional farmacêutico na avaliação das prescrições, visando o uso racional de medicamentos.

Palavras chave: interação medicamentosa, prescrição, Fisioterapia 
INTRODUÇÃO

O medicamento como recurso terapêutico pode apresentar várias peculiaridades. Com a descoberta de novas substâncias com melhores características que permitem o combate a problemas crônicos em uma população cada vez mais idosa, o emprego inadequado de medicamento pode provocar reações adversas, diminuindo a efetividade dos tratamentos medicamentosos já existentes (1).

Esses medicamentos podem interagir com outras moléculas, desde o seu preparo, por interações físico-químicas dos compostos da solução ou pela mistura em mesmo recipiente, até nas etapas de absorção, distribuição, metabolização e eliminação, acarretando modificações na sua concentração plasmática; ou na ligação ao receptor farmacológico, aumentando ou reduzindo seus efeitos farmacológicos. Dependo do local onde estiver ocorrendo interação farmacológica, esta poderá ser descrita como: farmacêutica, farmacocinética ou farmacodinâmica (2).

Quando é farmacodinâmica, os medicamentos podem causar efeitos que alteram bioquimicamente e fisiologicamente um organismo, modificando funções celulares. Tais efeitos podem ser mensuráveis e observados clinicamente. Podem ainda ocorrer alterações quando o medicamento é usado concomitantemente com outros fatores (dieta, exercício físico, horário da dose, associação medicamentosa) (3).

Nas interações medicamentosas podem ocorrer efeitos positivos ou negativos, ou seja, sinergismo, antagonismo parcial ou antagonismo total. Essas interações citadas não podem ser tarjadas definitivamente como benéficas ou nocivas, pois dependem de como é a terapêutica empregada (3).

As interações benéficas se traduzem por meio de sinergismo quando dois medicamentos juntos potencializam a terapêutica; ou por antagonismo, diminuindo efeitos indesejáveis de um medicamento. As interações nocivas causam efeitos adversos tanto por meio da sinergia quanto pelo antagonismo, causando diminuição do efeito terapêutico de um ou ambos os fármacos envolvidos (3).

Segundo Destruti (1999), a interação é definida como o uso concomitante de dois ou mais medicamentos (4). Essas interações podem alterar o efeito farmacológico, aumentando a eficácia terapêutica ou provocando reações adversas, sendo que sua ocorrência aumenta com o número de medicamentos em uso $(5,6)$.

Embora a incidência de interações medicamentosas seja mais alta nos idosos, por, geralmente, utilizarem diversos medicamentos, muitos pacientes pediátricos também apresentam esse perfil. Os pacientes que necessitam de tratamento fisioterápico, em qualquer faixa etária, não fogem à regra, podendo também ser sujeitos à ocorrência de polifarmacoterapia. Na Clínica-Escola de Fisioterapia da Universidade Paulista, Campus Sorocaba, que oferece atendimento a pacientes encaminhados pela rede pública, a ocorrência da utilização de mais de um medicamento é observada

Desse modo, o objetivo do presente trabalho foi investigar evidências de interação entre medicamentos pres- critos para pacientes de uma Clínica-Escola de Fisioterapia, no município de Sorocaba, SP.

\section{MATERIAIS E MÉTODOS}

Os dados foram coletados por meio da análise de prescrições médicas de pacientes de uma Clínica-Escola de Fisioterapia, no município de Sorocaba, SP. O estudo foi descritivo e quantitativo, envolvendo medicamentos listados nas prescrições de pacientes atendidos dentro das especialidades de Ortopedia e Neurologia.

A pesquisa ocorreu entre os meses de junho a setembro de 2015, totalizando 29 prescrições consultadas. Os pacientes que frequentaram a Clínica-Escola de Fisioterapia foram encaminhados pela Rede Pública de Saúde, recebendo acompanhamento dos alunos/estagiários e fisioterapeutas supervisores. Cabe ressaltar que apenas as prescrições foram avaliadas, não havendo acompanhamento dos pacientes.

O projeto foi aprovado pelo Comitê de Ética em Pesquisa (CEP) da Universidade Paulista, sob o número 1.103.946, aprovado no dia 11/06/2015.

\section{RESULTADOS E DISCUSSÃO}

Foram analisadas 29 prescrições de pacientes da Clínica Escola de Fisioterapia. Foi observado um total de 53 diferentes tipos de medicamentos, dentre eles, anti-inflamatórios, diuréticos, anti-hipertensivos, antidiabéticos, entre outros. Dentre as prescrições consultadas, foi constatado que prescrições contendo ácido acetilsalicílico apresentaram 14 potenciais interações. Essas interações foram baseadas nos dados da literatura e comparadas com os dados obtidos (Tabela 1).

Em relação aos medicamentos mais utilizados, os mais prescritos foram para tratamento da hipertensão, sendo os diuréticos tiazídicos e antagonistas dos receptores da angiotensina II os mais prevalentes. A segunda classe mais prescrita foi a de anti-inflamatórios não esteroidais (AINE), prevalecendo o ácido acetilsalicílico.

Tabela 1: Número de interações entre fármacos presentes em medicamentos prescritos para pacientes de uma Clínica-Escola de Fisioterapia, Sorocaba, SP, Brasil.

\begin{tabular}{|l|l|}
\hline fármacos & interaçőes \\
\hline ácido acetilsalicílico & 14 \\
\hline metformina & 12 \\
\hline hidroclorotiazida & 9 \\
\hline captopril & 6 \\
\hline furosemida & 5 \\
\hline levotiroxina & 4 \\
\hline glibenclamida & 4 \\
\hline insulina & 4 \\
\hline atenolol & 3 \\
\hline $\begin{array}{l}\text { enalapril } \\
\text { nimesulida }\end{array}$ & 3 \\
\hline
\end{tabular}


A faixa etária média dos pacientes foi 53 anos (mulheres) e 59 anos (homens) (Tabela 2). A média de medicamentos prescritos, por paciente, foi 3-4. A potencial ocorrência de polifarmacoterapia foi observada em prescrições de 7 pacientes, sendo 3 em prescrições de mulheres e 4 em prescrições de homens.

As interações mais frequentes foram entre as classes dos diuréticos e AINE, e hipoglicemiantes orais com betabloqueadores adrenérgicos. A frequência de interações está representada na Tabela 2.

Foram observadas combinações de medicamentos da classe dos anti-hipertensivos com predominância de associações de losartana e hidroclorotiazida, em $28,57 \%$ das prescrições analisadas.

Tabela 2: Classes terapêuticas envolvidas em interações medicamentosas potenciais em prescrições de pacientes atendidos em uma Clínica-Escola de Fisioterapia, Sorocaba, SP, Brasil.

\begin{tabular}{|c|c|c|}
\hline $\begin{array}{c}\text { Classe } \\
\text { terapêutica }\end{array}$ & $\begin{array}{c}\text { Frequência da } \\
\text { Interação }\end{array}$ & $(\%)$ \\
\hline $\begin{array}{l}\text { diuréticos } \\
\text { tiazídicos }\end{array}$ & 27 & 39,1 \\
\hline aine & 21 & 30,4 \\
\hline $\begin{array}{l}\text { hipoglicemiantes } \\
\text { orais }\end{array}$ & 19 & 27,5 \\
\hline $\begin{array}{c}\text { betabloqueadores } \\
\text { adrenérgicos }\end{array}$ & 13 & 18,8 \\
\hline
\end{tabular}

Os riscos resultantes das interações observadas podem ser desde desconforto gastrintestinal até insuficiência renal (Tabela 3).

Tabela 3: Efeitos das interações medicamentosas entre diferentes classes terapêuticas

\begin{tabular}{|c|c|}
\hline Classe terapêutica & Efeito/Risco \\
\hline $\begin{array}{l}\text { anti-hipertensivos+ } \\
\text { anti-hipertensivos }\end{array}$ & $\begin{array}{l}\text { podem causar queda da } \\
\text { pressão arterial }\end{array}$ \\
\hline $\begin{array}{l}\text { anti-hipertensivos+ } \\
\text { hipoglicemiantes }\end{array}$ & $\begin{array}{l}\text { aumento do efeito } \\
\text { hipoglicemiante }\end{array}$ \\
\hline $\begin{array}{l}\text { anti-hipertensivos } \\
\text { b-bloqueadores (seletivos ou } \\
\text { não)+diuréticos }\end{array}$ & $\begin{array}{l}\text { aumento do nível da glicemia } \\
\text { sanguínea }\end{array}$ \\
\hline $\begin{array}{l}\text { anti-hipertensivos+ } \\
\text { diuréticos }\end{array}$ & $\begin{array}{l}\text { dependendo da situação } \\
\text { patológica do indivíduo, pode } \\
\text { causar hipotensão }\end{array}$ \\
\hline $\begin{array}{l}\text { diuréticos+ } \\
\text { hipoglicemiantes }\end{array}$ & $\begin{array}{l}\text { diminuição do efeito } \\
\text { terapêutico de } \\
\text { hipoglicemiantes }\end{array}$ \\
\hline diuréticos+ AINE & insuficiência renal \\
\hline
\end{tabular}

Nas associações de losartana e/ou hidroclorotiazida com AINE pode ocorrer atenuação do efeito anti-hipertensivo, pelo mecanismo de inibição da síntese de prostaglandina renal, além do aumento de retenção de líquidos, induzida pelos AINE, promovendo interação medicamentosa negativa. Essas associações podem complicar ainda mais o quadro clínico de pacientes, pois essas interações poderão comprometer a função renal, pois os AINE inibem a síntese de prostaglandinas e os antagonistas de receptores de angiotensina II agravam a função renal por induzir vasoconstrição arteriolar e, consequentemente, diminuir a filtração glomerular (7).

Há um número considerável de pacientes que apresentam hipertensão e Diabetes mellitus. Esse resultado é corroborado por achados da literatura $(9,10,11,12,13)$.

O diabetes é uma doença multifatorial, que envolve fatores genéticos e comportamentais, como obesidade, sedentarismo e ingestão alimentar excessiva (8). Para as associações antidiabéticas foram constatadas: insulina + metformina e metformina + glibenclamida. Também foi observada uma preferência pela metformina no tratamento do diabetes.

Parece haver uma tendência de aumento na prevalência de Hipertensão Arterial e Diabetes mellitus $(14,15)$, refletindo na prevalência de uso de diuréticos, anti-hipertensivos e AINE, levando a um aumento de na ocorrência de interações medicamentosas envolvendo essas classes terapêuticas $(16,17,18)$.

Para avaliar as interações medicamentosas devem ser observados o risco potencial, a extensão e a significância clínica, sendo necessário considerar alguns fatores relacionados ao fármaco e ao paciente (predisposição genética, suscetibilidade a efeitos adversos, fatores epidemiológicos, etc.) $(19,20)$. Fatores importantes a serem considerados são a idade do paciente, as condições de sobrevivência e o local de habitação, pois estudos apontam que a polifamacoterapia é comum em adultos idosos e maior para aqueles que vivem em asilos, com consequências clínicas negativas (21).

$\mathrm{O}$ conhecimento das interações medicamentosas é fundamental para evitar o potencial risco à saúde do paciente, cabendo ao farmacêutico estar atento para os fármacos prescritos (12). Segundo Locatelli (2007), uma potencial interação medicamentosa pode ser prevista a partir do conhecimento das propriedades farmacológicas dos fármacos envolvidos, não necessariamente ocorrendo em todos os usuários (22). Portanto, deve ser avaliado se uma interação medicamentosa está causando alterações em parâmetros clínicos e/ou laboratoriais de determinado paciente para determinar a sua relevância clínica, caso a caso. Conhecer a natureza, o risco e a gravidade potencial da associação de um medicamento a outro é primordial na prescrição, dispensação e administração dos medicamentos. É necessário que o farmacêutico esteja integrado à equipe multiprofissional de saúde, pois sua presença é imprescindível para minimizar os erros frequentes em prescrições, fornecendo informações sobre os medicamentos aos demais profissionais de saúde, diminuindo riscos e garantindo uma melhora na qualidade ao atendimento do paciente $(23,24)$.

Além de todos esses fatores importantes relacionados ao papel do farmacêutico, cabe ressaltar que as in- 
tervenções farmacêuticas resultam não só em melhora do paciente, mas, também, em considerável redução de custos para as instituições, pois quando os pacientes internados são expostos à polifarmacoterapia apresentam grande potencial de problemas no tratamento, gerando novos tratamentos e novos custos $(25,26)$.

A Organização Mundial da Saúde (OMS) orienta que todo farmacêutico disponha ao paciente ao menos 3 minutos para orientações. Esse tempo é fundamental para que o profissional da saúde informe ao paciente a importância do cumprimento da dosagem, a influência dos alimentos, as interações medicamentosas, as possíveis reações adversas e a melhor maneira de se conservar o medicamento

\section{CONCLUSÃO}

Com relação a esse estudo, as principais limitações estão relacionadas ao fato de não ter sido possível avaliar

\section{REFERÊNCIAS}

1. Kawano DF, Pereira LRL, Ueta JM, Freitas O. Acidentes com os medicamentos: como minimizá-los. 2006; Braz J. Pharm Sci. 42:487-495.

2. Secoli SR. Interações medicamentosa: fundamentos para a prática clínica de enfermagem. Rev Esc Enf Usp. 2001; 35:28-34.

3. Fuchs FD, Wannmacher L. Farmacología clínica: Fundamentos da Terapêutica Racional. $4^{\circ}$ ed., 2012.

4. Destruti ABCB. Interações medicamentosas. 2. Ed. São Paulo, SENAC; 1999.58 p.

5. Bisson MP. Farmácia Clínica \& atenção farmacêutica. 2.Ed. São Paulo: Manole, 2007. 371p.

6. Hartshorn EA. Drug Interaction. Ann Pharmacother 2006; 40(1): 112-113.

7. Drugs interactions between aspirin low strength and hydrochlorothiazide/losartan. Disponível em: http://www. drugs.com/drug-interactions/aspirin-low-strength-with-hydrochlorothiazide-losartan-243-3197-1260-0.ht$\mathrm{ml}$ ?professional=1. Acesso em 10/09/2015.

8. SBD. Sociedade Brasileira de Diabetes, Diretrizes Brasileiras De Diabetes, 2009. Disponível em: http://www.proac. uff.br/farmacoclinica/sites/default/files/diretrizes09_final_0.pdf. Acesso em 12/09/2015.

9. Veronez LL, Simões MJS. Análise da prescrição de medicamentos de pacientes hipertensos atendidos pelo SUS da rede municipal de Saúde de Rincão - SP. Rev Ciênc Farmac Basica Apl, 2009; 29(1):45-51.

10. Tavares MS, Macedo TC, Mendes DRG. Potential drug interactions in a group of patients with hypertension and diabetes of the family health strategy. Rev Div Cient Sena Aires, 2012; 2:119-126.

11. Ribeiro M, Faria L, Lemos G. Atenção farmacêutica em pacientes com hipertensão arterial sistêmica em uma Unidade de Saúde de Jequié-BA. J Manag Prim Health Care. 2013. 4(3):176-182.

12. Almeida ER, Moutinho CB, Leite MTS. A prática da educação em saúde na percepção dos usuários hipertensos e a relevância clínica de cada interação detectada, devido à falta de acesso a outros dados dos pacientes como exames laboratoriais e história clínica mais apurada.

Por fim, vale ressaltar a necessidade do profissional farmacêutico no acompanhamento dos pacientes em polifarmacoterapia para evitar uma possível interação prejudicial, visando ao uso racional de medicamentos, que contribui para a melhoria na qualidade de vida do paciente.

\section{AGRADECIMENTOS}

Os autores agradecem à Profa. Dra. Beatriz de Oliveira Peixoto pela autorização no contato e acesso às prescrições dos pacientes da Clínica-Escola de Fisioterapia, à Universidade Paulista, Campus Sorocaba, pela oportunidade no desenvolvimento do trabalho e ao Prof. Me. Ricardo José Orsi de Sanctis pela revisão gramatical.

diabéticos. Saúde Debate. 2014; 38 (101):328-337. DOI: 10.5935/0103-1104.20140030.

13. Monteiro SCM, Belfort IKP, Sousal WR, Barros CS, Campos KVS. Estudo de potencias interações medicamentosas em pacientes hipertensos. Infarma, 2015; 27(2):117125. DOI: $10.14450 / 2318-9312$.

14. Amaral DMD, Perassolo MS. Possíveis Interações medicamentosas entre os anti-hipertensivos e antidiabéticos em participante do Grupo HIPERDIA de Parobé, RS (Uma análise teórica). Rev. Ciênc Farm Básica Apl. 2012; 33(1):99-105.

15. Lima RF, Machado AV, Rebelo FM, Naves JOS, Lavich TR, Dullius J. Interações medicamentosas potenciais em diabéticos tipo 2 participantes de um programa de educação em saúde. Infarma. 2015; 27(3):160-167. DOI: 10.14450/2318-9312.

16. Sehn R, Camargo AL, Heineck I, Ferreira MBC. Interações medicamentosas potencias em prescrições de pacientes hospitalizados. Infarma, 2003; 15(9-10):77-81.

17. Santos JC, Faria Junior M, Restini CBA. Potenciais interações medicamentosas identificadas em prescrições a pacientes hipertensos. Rev Bras Clin Med. São Paulo. 2012; 10(4):308-317.

18. Nascimento DM, Pigoso AA. Interação Medicamentosa entre anti-hipertensivos e anti-inflamatórios não esteroides. Rev Cient FHO Uniararas. 2013; 1(1): 14-17.

19. Maher RL, Hanlon J, Hajjar ER. Clinical consequences of polypharmacy in elderly. Expert Opin Drug Safety 2014; 13(1):57-65. DOI: 10.1517/14740338.2013.827660.

20. Janchawee B, Wongpoowarak W, Owatranporn T, Chongsuvivatwong V. Pharmacoepidemiologic study of potential drug interactions in outpatients of a university hospital in Thailand. J Clin Pharm Ther 2005; 30:13-20. DOI: 10.1111/j.1365-2710.2004.00598.x.

21. Hoefler R, Wannacher L. Interações de medicamentos. In: Uso racional de Medicamentos, Temas Selecionados. Brasil. Ministério da Saúde/ OPAS. Disponível em: http:// 


\section{lortarma}

www.paho.org/bra/index.php?gid=1316\&option=com docman\&task=doc_view. Acesso em 15/09/2015.

22. Locatelli J. Interações medicamentosas em idosos hospitalizados. Einstein, 2007; 5(4):343-346.

23. Queiroz KCB, Nascimento MFS, Fernandes V, Miotto FA. Análise de Interações Medicamentosas Identificadas em Prescrições da UTI Neonatal da ICU-HGU. UNOPAR Cient Ciênc Biol Saúde 2014; 16(3):203-207.
24. Ansari JA. Drug interaction and pharmacist. J Young Pharm 2010; 2(3):326-331. DOI: 10.4103/0975-1483.66807.

25. Jameson JP, VanNoord GR. Pharmacotherapy consultation on polypharmacy patients in ambulatory care. Ann Pharmacother 2001; 35(7-8):835-840.

26. Chumney EC, Robinson LC. The effects of pharmacist interventions on patients with polypharmacy. Pharm Practice 2006; 4(3):103-109 